\title{
Differential Proteomic Analysis of Syncytiotrophoblast Extracellular Vesicles from Early-Onset Severe Preeclampsia, using 8-Plex iTRAQ Labeling Coupled with 2D Nano LC-MS/MS
}

\author{
Hongmei Lia Lei Han ${ }^{a, b}$ Zhiling Yanga Wei Huang ${ }^{a}$ Xin Zhang ${ }^{a}$ Yan Gu \\ Yinfeng Lia Xiaojie Liu ${ }^{a}$ Lijuan Zhou ${ }^{a}$ Jiongyu Huc Meijia Yu ${ }^{d}$ Jing Yang ${ }^{e}$ Yilin Li $^{f}$ \\ Yingru Zheng ${ }^{a}$ Jianxin Guo Jian Han ${ }^{a} \quad \mathrm{Li} \mathrm{Li}^{\mathrm{a}}$ \\ aDepartment of Obstetrics and Gynecology, Research Institute of Surgery, Daping Hospital, Third \\ Military Medical University, Chongqing, ${ }^{b}$ Department of Obstetrics and Gynecology, the 306th Hospital \\ of the Chinese People's Liberation Army, Beijing, 'Department of Endocrinology and Metabolism, \\ Southwest Hospital, Third Military Medical University, Chongqing, dDepartment of Obstetrics and \\ Gynecology, Southwest Hospital, Third Military Medical University, Chongqing, eDepartment of \\ Endocrinology, Xinqiao Hospital, Third Military Medical University, Chongqing, 'Department of \\ Obstetrics and Gynecology, Sichuan Academy of Medical Science and Sichuan Provincial People's \\ Hospital, Chendu, China
}

\section{Key Words}

Preeclampsia - Syncytiotrophoblast extracellular vesicles - Microvesicles - Microparticles • Exosomes • iTRAQ • Proteomics

\begin{abstract}
Aims: Previous studies have revealed that the increased shedding of syncytiotrophoblast extracellular vesicles (STBM) may lead to preeclampsia (PE). We aimed to identify the proteins carried by STBM and their potential pathological roles in early-onset severe PE. Methods: In this study, we performed a differential proteomic analysis of STBM from early-onset severe PE patients, using iTRAQ isobaric tags and 2D nano LC-MS/MS. STBM were generated by the in vitro explant culture method, and then verified by electron microscopy and western blot analysis. Results: A total of 18533 unique peptides and 3317 proteins were identified, 3292 proteins were quantified. We identified 194 differentially expressed proteins in STBM from early-onset severe PE patients, 122 proteins were up-regulated and 72 proteins were downregulated. Further bioinformatics analysis revealed that mitochondrion, transmembrane transport and transmembrane transporter activity were the most abundant categories in gene ontology (GO) annotation. Glycolysis/ gluconeogenesis, citrate cycle, fatty acid elongation, steroid hormone biosynthesis and oxidative phosphorylation were the five significantly


represented pathways. Four differentially expressed proteins (siglec-6, calnexin, CD63 and S100-A8) related to inflammation, coagulation or immunoregulation were independently verified using western blot. Conclusions: The identification of key proteins carried by STBM may serve not only as a basis for better understanding and further exploring the etiology and pathogenesis of $\mathrm{PE}$, but also as potential biomarkers and in providing targets for future therapy in $\mathrm{PE}$, especially in early-onset severe $\mathrm{PE}(\mathrm{SPE})$.

Copyright $\odot 2015$ S. Karger AG, Basel

\section{Introduction}

Preeclampsia (PE) is a pregnancy-specific disease characterized by hypertension and proteinuria after 20 weeks gestation, which affects $2-8 \%$ of pregnancies worldwide. Earlyonset $\mathrm{PE}$ ( $<34$ weeks' gestation) is generally regarded as a severe condition, and is a leading cause of maternal and neonatal mortality, especially in developing countries [1].

As the pathogenesis of PE remains not well illuminated, the therapy is passive and limited, and the termination of pregnancy is the only definitive treatment. Severe PE(SPE) was defined as blood pressure $\geq 160 / 110 \mathrm{mmHg}$, heavy proteinuria $(\geq 3+)$ and manifestations of multiple organ damage or dysfunction [2]. Consequently, sPE is an important iatrogenic cause of prematurity. Recently the placenta has become a focus of researchers' attention, as early-onset PE is generally considered as a primarily placental disease [3-5]. Syncytiotrophoblast extracellular vesicles (STBM) are continuously shed and secreted from the placental syncytium into the maternal circulation in normal pregnancy. Significantly increased amounts of STBM have been discovered in PE [6], especially in early-onset PE [7, 8]. STBM mainly comprise microparticles and exosomes, which vary in size, morphology and function. Quantities of in vitro experiments focused on the biological functions of STBM in PE have uncovered proinflammatory [9-12], procoagulant [13, 14], anti-angiogenic [15-21] and immunoregulatory activities [10,22-25], which may explain the principal pathophysiological processes and clinical features of this disorder. However, the key molecules, as well as the precise mechanism of STBM in PE, are still not fully understood. As proteins are the molecules that execute a vast array of functions, there may be multiple proteins carried by STBM involved the aforementioned processes. Analysis of the composition of STBM and its essential proteins may result in targets for intervention and blockade that providing new therapeutic strategies. To our knowledge, only one review article [26] from a research team at the University of Oxford has mentioned their partial STBM proteomics results. To uncover the total protein composition of STBM, further studies are required.

Isobaric tags for relative and absolute quantitation (iTRAQ) combined with twodimensional liquid chromatography-tandem mass spectrometry (2D LC-MS/MS) is one of the most powerful methodologies in quantitative proteomics. Here, we aim to establish a comparative proteome profile of the STBM in normal and early-onset PE pregnancies using 8-Plex iTRAQ labeling coupled with 2D nano LC-MS/MS.

\section{Materials and Methods}

Patients

The study protocol was approved by the Research Ethics Committee of the Daping Hospital (Chongqing, China). All 36 participants provided written informed consent according to the Declaration of Helsinki. Human placentae were obtained sterile after caesarean delivery, all samples were processed within 30 min of collection. Control pregnant women were from breech presentation or scarred uterus pregnancy, and selected if they had no history of hypertension or chronic illness, a singleton pregnancy without known fetal abnormalities, and natural conception. Early-onset sPE was defined as new hypertension (blood pressure $\geq 160 / 110 \mathrm{~mm} \mathrm{Hg}$ on two consecutive occasions) and new proteinuria ( $24 \mathrm{~h}$ secretion of $\geq 5 \mathrm{~g}$ or $\geq 3+$ ), in the absence of urinary tract infection. Women with chronic hypertension, chronic renal disease, diabetes mellitus, cardiac disease, or autoimmune disease were excluded from the study. 


\section{Purfication of STBM}

STBM were prepared from placentae by modification of the method of in vitro explant culture, as previously described [15, 27]. Briefly, the villous tissue was isolated from the placentae by carefully removing the decidua and visible vessels, washing six times in sterile phosphate buffered saline (PBS), and then cutting the villous tissue into 1- to 2-mm pieces. Two hundred milligram wet weight of explants were cultured in $100 \mathrm{~mm}$ culture dishes (Corning, NY) with $30 \mathrm{ml}$ of Dulbecco Modified Eagle's Medium (DMEM): F12 Nutrient Mixture (1:1) (Gibco, Grand Island, NY) containing 1\% antimycotic/antibiotics (Gibco, Grand Island, NY), 10\% fetal bovine serum (Gibco, Grand Island, NY), $25 \mathrm{U} / \mathrm{ml}$ heparin (Roche Diagnostics, Germany) and $50 \mathrm{U} / \mathrm{ml}$ aprotinin (Sigma, USA) for $72 \mathrm{~h}$ at $37^{\circ} \mathrm{C}$. To purify STBM, culture supernatants were processed by a four-step centrifugation/ultracentrifugation at $4^{\circ} \mathrm{C}$ (by modification of the method of Smith [28] and Théry [29]): $1000 \times \mathrm{g}$ for $15 \mathrm{~min} ; 10000 \times \mathrm{g}$ for $15 \mathrm{~min}$, at each of these steps, the pellet (include large dead cells and large cell debris) is thrown away, and the supernatant is used for the following step, at the third step the supernatant is ultracentrifugation at $100000 \times \mathrm{g}$ for $60 \mathrm{~min}$, then the pellets were pooled and washed in a large volume of sterile $0.22 \mu \mathrm{M}$ filtered phosphate buffered saline (fPBS), and then ultracentrifuged at $1000000 \times \mathrm{g}$ for another $60 \mathrm{~min}$ to eliminate contaminating proteins, the final pellets were resuspended in $\mathrm{fPBS}$, aliquoted and stored at $-80^{\circ} \mathrm{C}$ until used. Total protein concentrations were measured by a bicinchoninic acid (BCA) assay (Beyotime Biotechnology, Haimen, China).

\section{Electron microscopy (EM)}

Scanning electron microscopy. STBM suspensions were mixed 1:1 with 2.5\% glutaraldehyde for $2 \mathrm{~h}$ at room temperature. One drop of fixed STBM suspension was deposited on mica slice (pretreated with 95\% alcohol), covered and allowed to dry. After gold-plating surface treatment, the preparations were examined and photographed in a scanning electron microscope (SEM; Hatchi, S-3400N II, Japan). STBM diameters were measured using Image-Pro Plus 6.0 software.

Immunoelectron Microscopy. STBM suspensions were mixed 1:1 with 4\% paraformaldehyde for 30 $\min$ at $4^{\circ} \mathrm{C}$ and then were applied to 200 -mesh nickel grids, treated with PBS/50 mM glycine and 5\% bovine serum albumin(BSA)/10\% goat serum to block non-specific binding, then incubated with NDOG1(Abcam, ab11460) diluted $1: 100$ in 1\% BSA-PBS blocking solution for $60 \mathrm{~min}$ at $4^{\circ} \mathrm{C}$. After washing by $0.1 \% \mathrm{BSA}-$ PBS and 0.5\%BSA-PBS in turn, the grids were incubated with $1.4 \mathrm{~nm}$ NANOGOLD ${ }^{\circledR}$ goat anti-mouse IgG (Nanoprobes, USA) for $30 \mathrm{~min}$ at $4^{\circ} \mathrm{C}$, while the control group were incubated with $1 \%$ BSA-PBS instead of goat anti-mouse IgG. Grids were then transferred to $2.5 \%$ glutaraldehyde for 5 min to stabilize the immunoreactions. Subsequently, the grids were incubated with silver enhance solution (Nanoprobes, USA) for $5 \mathrm{~min}$. After rinsing with PBS and distilled water, the grids were negatively stained with uranyl acetate and examined in the transmission electron microscope (TEM; Philips, TECNAI10, Holland) at $80 \mathrm{kV}$.

\section{Western blotting}

Western blotting was performed to assess the preparation of STBM and verify the differentially expressed proteins by iTRAQ analysis. One-hundred microliters of STBM suspension was lysed by $100 \mu \mathrm{L} 1 \times$ RIPA buffer containing $1 \mathrm{mM}$ PMSF. Lysates were centrifuged at $12000 \times \mathrm{g}$ for $15 \mathrm{~min}$ at $4^{\circ} \mathrm{C}$. Protein levels were quantified by the BCA method. After boiling in SDS-PAGE sample buffer, the proteins were separated with $10 \%$ or $15 \%$ SDS-PAGE gels and then transferred to PVDF membranes (Millipore, Billerica, MA, USA). After blocking with TBST (50 mM Tris- $\mathrm{HCl}$ pH 7.4, $0.5 \mathrm{M} \mathrm{NaCl}, 0.05 \%$ Tween 20) containing 5\% (w/v) skim milk for $4 \mathrm{~h}$ at $37^{\circ} \mathrm{C}$, the membranes were incubated overnight at $4^{\circ} \mathrm{C}$ with primary antibodies to hsp70 (Thermo Fisher, MA, PA5-28003, 1:500), tsg101 (Santa Cruz, CA, sc7964, 1:1000), flotillin-1 (Santa Cruz, CA, sc25506, 1:500), siglec-6 (Abcam, MA, ab38581, 1:800), Calnexin (Cell Signaling Technology, MA, 2679,1:1000), CD63 (Abcam, MA, ab118307,1:800), and S100-A8 (Abcam, MA, ab92331, 1:2000). After three washes with TBST, membranes were incubated with the appropriate secondary antibody (1: 2000) at room temperature for $2 \mathrm{~h}$, followed by incubation in enhanced chemiluminescence (ECL) kit (GE Healthcare, USA). The proteins were quantified using Labwork 4.6 (UVP, Upland, CA, USA). The immunoblot experiments were repeated at least three times, and representative images are shown.

Trypsin Digestion and iTRAQ Isobaric Labeling

Protein digestion, peptide extraction, and LC-MS/MS analysis were performed as described previously $[30,31]$. Briefly, each sample of proteins $(300 \mu \mathrm{g})$ was added with dithiotreitol solution diluted in $100 \mathrm{mM}$, 


\section{Cellular Physiology Cell Physiol Biochem 2015;36:1116-1130 \begin{tabular}{l|l} 
and Biochemistry 10.1159/000430283 & $\begin{array}{l}\text { O 2015 S. Karger AG, Basel } \\
\text { www.karger.com/cpb }\end{array}$ \\
\cline { 2 - 3 }
\end{tabular}}

Li et al.: Proteomic Analysis of Syncytiotrophoblast Extracellular Vesicles

incubated in boiling water for $5 \mathrm{~min}$, cooled to room temperature, then mixed with $200 \mu \mathrm{L}$ UA buffer (8 M Urea and $150 \mathrm{mM}$ Tris-HCl pH8.0) and transferred onto a $10 \mathrm{kD}$ ultrafiltration filter (Sartorius, German), centrifuged at $14,000 \times \mathrm{g}$ for $15 \mathrm{~min}$, mixed and centrifuged again with $200 \mu \mathrm{L}$ UA buffer. The filter solution was then discarded. Subsequently, $100 \mu \mathrm{L}$ of iodoacetamide (50 mM in UA buffer) was added into the filter, oscillated at $600 \times \mathrm{g}$ for $1 \mathrm{~min}$. After $30 \mathrm{~min}$ of incubation in darkness, the filter was centrifuged at 14000 $\times \mathrm{g}$ for $10 \mathrm{~min}$, washed twice with $100 \mu \mathrm{L}$ UA buffer as the previous condition. Then, $100 \mu \mathrm{L}$ dissolution buffer (Applied Biosystems, USA) was added to the filter and centrifuged as the previous condition for twice. Finally, $5 \mu \mathrm{g}$ trypsin in $40 \mu \mathrm{L}$ dissolution buffer was added to each filter, oscillated at $600 \times \mathrm{g}$ for $1 \mathrm{~min}$, incubated at $37^{\circ} \mathrm{C}$ for $16-18 \mathrm{~h}$, then the filter was transferred to a new collecting pipe and centrifuged at 14 $000 \times \mathrm{g}$ for $10 \mathrm{~min}$. The peptide concentration was then analyzed at $280 \mathrm{~nm}$ [31].

Fifty micrograms of peptide mixture was labeled with the 8-plex iTRAQ reagents according to the manufacturer's protocol (Applied Biosystems, USA). For the iTRAQ experiments, three independent biological replicates and two technical replicates were performed in each group. In sPE group: biological replicate 1 was labeled 117, replicate 2 was labeled 118, replicate 3 was labeled 121, and technical replicates were labeled 117 and 119. In the control group: biological replicate 1 was labeled 113, replicate 2 was labeled 114, replicate 3 was labeled 115, and technical replicates were labeled 114 and 116. The three biological replicates were used to profile and quantitate the STBM proteome. The two technical replicates were used to detect the efficacy of mass spectrometer. The labeling solution reaction was incubated at room temperature for $1 \mathrm{~h}$ before further analysis.

\section{$2 D L C-M S / M S$}

Strong Cationic exchange Chromatography Separation. The mixed peptides were fractionated by strong cation exchange (SCX) chromatography on a Polysulfethyl $4.6 \times 100 \mathrm{~mm}$ column $(5 \mu \mathrm{m}, 200 \AA$, Poly LC Inc, Maryland, USA). Buffer A was composed of $10 \mathrm{mM} \mathrm{KH}_{2} \mathrm{PO}_{4} \mathrm{pH} 3.0$ and 25\% (v/v) acetonitrile, buffer B was buffer A with $500 \mathrm{mM} \mathrm{KCl}$.The peptides were eluted at a flow rate of $1 \mathrm{~mL} / \mathrm{min}$ with a gradient of $10-20 \%$ buffer B for $10 \mathrm{~min}, 20-45 \%$ buffer B for $10 \mathrm{~min}$. A total of 30 SCX fractions were collected, then combined into 8 pools and desalted on C18 cartridges (66872-U, Sigma).

Reversed Phase LC-ESI-MS/MS

Each SCX fraction was analyzed on a Q Exactive MS (Thermo Finnigan) equipped with Easy nLC (Thermo Fisher Scientific). Peptide mixture was separated by the Zorbax 300SB-C18 peptide traps (AgilentTechnologies, USA) $(100 \mathrm{~mm} \times 75 \mu \mathrm{m}, 3 \mu \mathrm{m})$ at $250 \mathrm{~nL} / \mathrm{min}$, then separated with a linear gradient of buffer C (84\% acetonitrile and $0.1 \%$ Formic acid) over $120 \mathrm{~min}$. MS/MS data were acquired using the top 10 most abundant precursor ions from the survey scan (300-1800 m/z) for HCD (high-energy collisional dissociation) fragmentation. Determination of the target value was based on predictive Automatic Gain Control (pAGC). The dynamic exclusion was $40.0 \mathrm{~s}$. The resolution was set to $70000 \mathrm{at} \mathrm{m/z} 200$ for survey scan and 17,500 at m/z 200 for HCD spectra, respectively. The normalized collision energy was $30 \mathrm{eV}$ and the underfill ratio was $0.1 \%$. The instrument was run with peptide recognition mode.

Data analysis

MS/MS spectra were performed using MASCOT engine (version 2.2, Matrix Science) embedded into Proteome Discoverer 1.4 (Thermo Electron, San Jose, CA, USA), searching against a human sequence database (uniprot_human.fasta, download January 2014, 134919 sequences). Search parameters were set as follows: peptide mass tolerance at $\pm 20 \mathrm{ppm}$, fragment mass tolerance at $0.1 \mathrm{Da}$, trypsin enzyme with the max missed cleavages up to 2, fixed modification of iTRAQ 8plex (N-term), iTRAQ 8plex (K), variable modification of oxidation (M) and iTRAQ 8plex (Y). All data were reported based on $99 \%$ confidence for protein and peptides identification as determined by false discovery rate (FDR) $\leq 1 \%$. Only spectra where all the expected ITRAQ reporter ions were detected and unique peptide were used for quantification. The Proteome Discoverer application calculated protein ratios as the median of all peptide hits belonging to a protein. The final ratios were then normalized with the median average protein ratio, assuming that most proteins remained unchanged in abundance.

Bioinformatics analysis

The cellular component, molecular function, and biological process of proteins were annotated by gene ontology (GO) database (http://www .geneontology.org/). The signaling pathways were generated by KARGER 


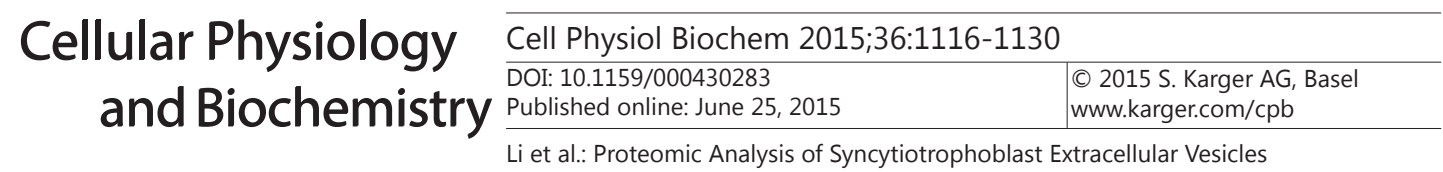

searching against the Kyoto Encyclopedia of Genes and Genomes database (http://www.genome.jp/kegg/ pathway.html). The protein-protein interaction network was analyzed by Search Tool for the Retrieval of Interacting Genes/Proteins (STRING) software (http://string.embl.de/). Hierarchical cluster analysis was performed on the log-transformed fold-change expression values for the differentially expressed proteins using the Cluster 3.0 software.

\section{Statistical analysis}

The experimental data were presented as means \pm standard deviation (SD). Differences between the control and SPE groups were analyzed with independent sample t tests using SPSS 18.0 software, for ITRAQ analysis, differentially expressed proteins were defined as $p<0.05$ and fold-change $\geq 1.2$, and significance was defined as $\mathrm{p}<0.05$ to calculate all other statistics.

\section{Results}

\section{Patient Data}

A total of 6 subjects were selected for the iTRAQ experiments, 3 participants per group (Table 1 upper section). The clinical characteristics of each group are presented in Table 1. There was no significant difference in parity (not shown) or maternal age at the time of booking. Body mass index and maximum diastolic and systolic blood pressures were all significantly higher in the PE patients compared to the control group (Table 1 upper section). As would be expected, birth weight and placenta weight were both significantly lower in sPE-affected pregnancies (Table 1 upper section). The pregnancy duration of the sPE group $(\mathrm{n}=3$ ) was no significant difference than that of the control group ( $233.7 \pm 2.1$ days vs 250.3 \pm 11.5 days, $p>0.05$ ). To verify the proteins detected by iTRAQ thirty additional subjects divided over the above groups were additionally included to enhance the numbers in the experiments, resulting in a total of 36 participating subjects (Table 1 lower section). The pregnancy duration of the $\mathrm{sPE}$ group $(\mathrm{n}=24)$ was no significant difference than that of the control group ( $231 \pm 6.9$ days vs $265 \pm 8.7$ days, $p>0.05$ ) as well.

\section{Validation of STBM using electron microscopy and Western blotting}

STBM was obtained by the combination of in vitro explant culture method and a four-step centrifugation/ultracentrifugation. STBM preparations were verified by EM and western blot analysis as shown in Figure 1. SEM showed that purified STBM were membrane-bound, irregular or spherical structures and heterogeneous in size, ranging from approximately

Table 1. Characteristics of the participating subjects

\begin{tabular}{|c|c|c|c|c|c|c|}
\hline \multirow{2}{*}{ Subjects participating in the iTRAQ study } & \multicolumn{3}{|c|}{ Control group } & \multicolumn{3}{|c|}{ sPE group } \\
\hline & & & & & & \\
\hline Sample name & 1 & 2 & 3 & 1 & 2 & 3 \\
\hline $\operatorname{Age}(y)$ & 22 & 27 & 22 & 26 & 31 & 26 \\
\hline BMI & 24.4 & 26.2 & 23.2 & 30.4 & 32.0 & 26.8 \\
\hline Gestation(days) & 257 & 257 & 237 & 233 & 232 & 236 \\
\hline Maximum systolic BP(mmHg) & 120 & 109 & 96 & 198 & 162 & 165 \\
\hline Maximum diastolic $\mathrm{BP}(\mathrm{mmHg})$ & 70 & 74 & 58 & 127 & 97 & 105 \\
\hline Proteinuria & - & - & - & +++ & +++ & +++ \\
\hline Birth weight (kg) & 3500 & 2950 & 2800 & 1357 & 1450 & 1520 \\
\hline Placenta weight $(\mathrm{kg})$ & 500 & 500 & 500 & 350 & 380 & 300 \\
\hline \multicolumn{7}{|c|}{ Subjects participating in the western blot study } \\
\hline Sample number & \multicolumn{3}{|c|}{$\mathrm{n}=12$} & \multicolumn{3}{|c|}{$n=24$} \\
\hline $\operatorname{Age}(y)$ & \multicolumn{3}{|c|}{$32.2 \pm 2.1$} & \multicolumn{3}{|c|}{$31.6 \pm 4.6$} \\
\hline BMI & \multicolumn{3}{|c|}{$23.8 \pm 1.9$} & \multicolumn{3}{|c|}{$29.4 \pm 3.0^{*}$} \\
\hline Gestation(days) & \multicolumn{3}{|c|}{$265 \pm 8.7$} & \multicolumn{3}{|c|}{$231 \pm 6.9^{*}$} \\
\hline Maximum systolic BP(mmHg) & \multicolumn{3}{|c|}{$112.8 \pm 10.4$} & \multicolumn{3}{|c|}{$172.4 \pm 10.7^{*}$} \\
\hline Maximum diastolic $\mathrm{BP}(\mathrm{mmHg})$ & \multirow{2}{*}{\multicolumn{3}{|c|}{$75.3 \pm 6.9$}} & \multicolumn{3}{|c|}{$119.7 \pm 8.6^{*}$} \\
\hline Proteinuria & & & & \multicolumn{3}{|c|}{+++} \\
\hline
\end{tabular}




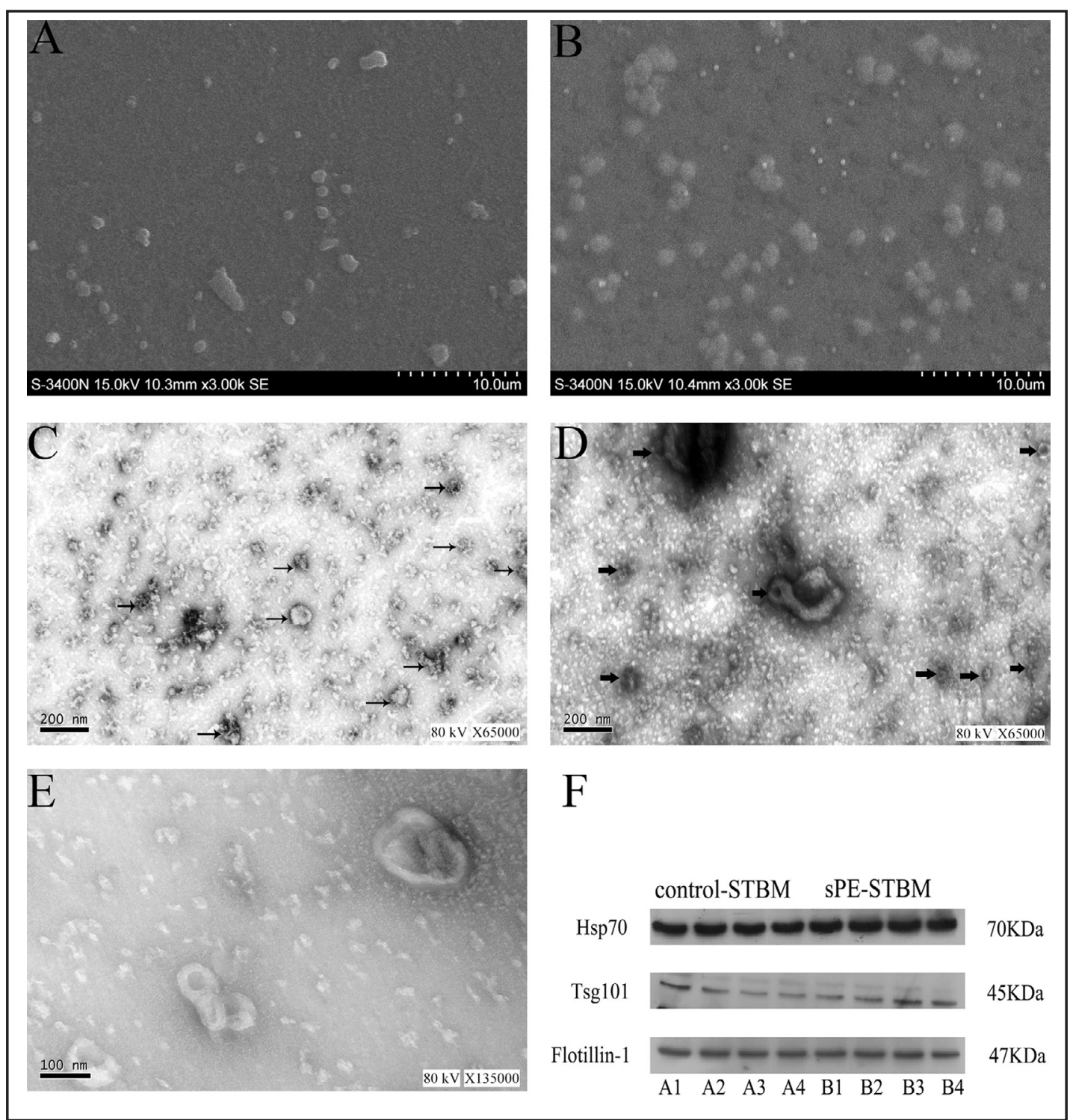

Fig. 1. Validation of STBM. (A) and (B) Representative scanning electron micrograph of STBM isolated from control and sPE group. (C) and (D) Representative transmission electron micrograph of STBM, immunogold labeling with anti-NDOG1(1:100). The arrowheads indicate labeled STBM. (E) Representative transmission electron micrograph of control group (incubated with 1\%BSA-PBS instead of goat anti-mouse IgG). (F) Representative image of western blot for the presence of hsp70, tsg101 and flotillin-1. A1-A4 and B1-B4 represent different STBM isolated from control and SPE group, respectively. A2 and A4, B1 and B3 are the same subjects in each group.

30 to $1700 \mathrm{~nm}$ (Fig. 1A, 1B). The morphology and size distribution were consistent with previously reported STBM preparation [15]. Western blots were performed to detect the marker proteins, with all the STBM fraction showing activity to EV biomarkers including hsp70, tsg101 and flotillin-1 as shown in Figure 1F. To confirm that syncytiotrophoblast membraneous material was present in the STBM preparations, we examined this for the presence of NDOG1, a syncytiotrophoblast-specific protein, using an immunoelectron microscopy. As observed in Figure 1C and Figure 1D, we confirmed that the NDOG1 antibody bound to placental vesicles by TEM, while there had no evident immunogold labeling in 


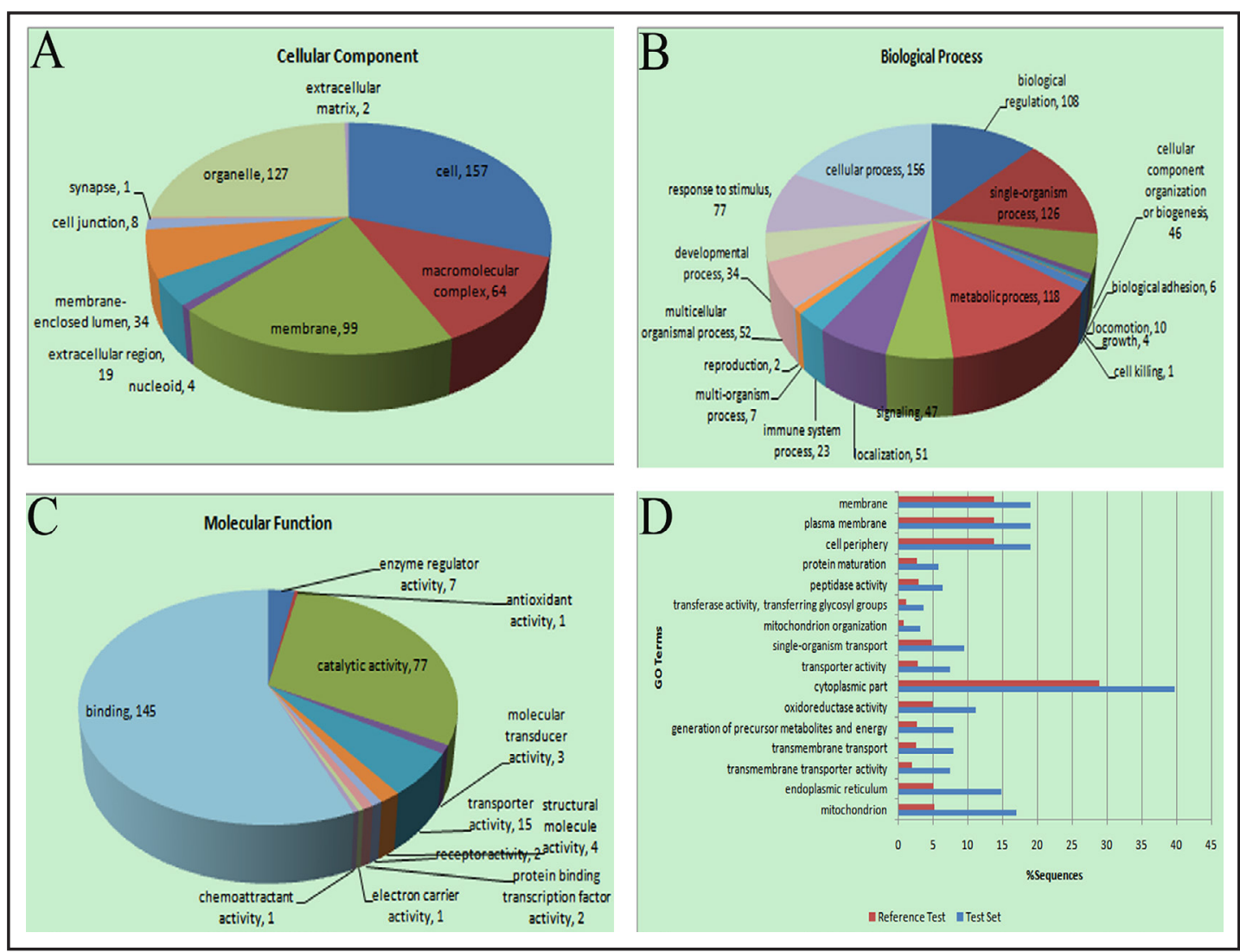

Fig. 2. Gene ontology annotation of differentially expressed proteins. (A) Cellular component. Allocation of 194 differentially expressed proteins by cell component showed that the majority belonged to the cell (157) and organelle (127), whereas the minority resided in the synapse (1). (B) Biological process. Allocation of differentially expressed proteins by biological process demonstrated that the greatest number of these proteins (156) was allocated to cellular process and the smallest (1) to cell killing. (C) Molecular function. Allocation of differentially expressed proteins by molecular function indicated that the highest number of proteins (311) was associated with binding activities and the lowest (1 each) with antioxidant activity, chemoattractant activity and electron carrier activity. (D) GO enrichment demonstrated that mitochondrion, transmembrane transport and transmembrane transporter activity were the most abundant categories in CC, BP and MF, respectively.

the control group (Fig. 1E). Taken together, these results suggest that STBM preparation consisted of highly enriched STBM from placentae by in the vitro explant culture method.

\section{Protein identification}

A total of 18533 unique peptides and 3317 proteins were identified, 3292 proteins were quantified in all eight label channels, the label rate of iTRAQ was $99.25 \%$. In the present study, proteins identified as differentially expressed had to meet the following criteria: peptides $\geq 1$; FDR $\leq 1 \%$; $p<0.05$; fold-change $\geq 1.2$ or $\leq 0.833$. As a result, 122 proteins were found to be upregulated and 72 were found to be downregulated in the STBM of SPE group compared with the control group (data not shown).

\section{Bioinformatics analysis}

Of the 194 differentially expressed proteins, 189 were classified using GO annotation. As shown in Figure 2, the cellular component (CC) of these proteins was mainly located in cell (157 proteins), organelle (127 proteins), membrane (99 proteins), macromolecular

\section{KARGER}


Fig. 3. KEGG pathway enrichment of 194 differentially expressed proteins. KEGG pathway enrichment revealed that the most salient pathway is glycolysis/gluconeogenesis.

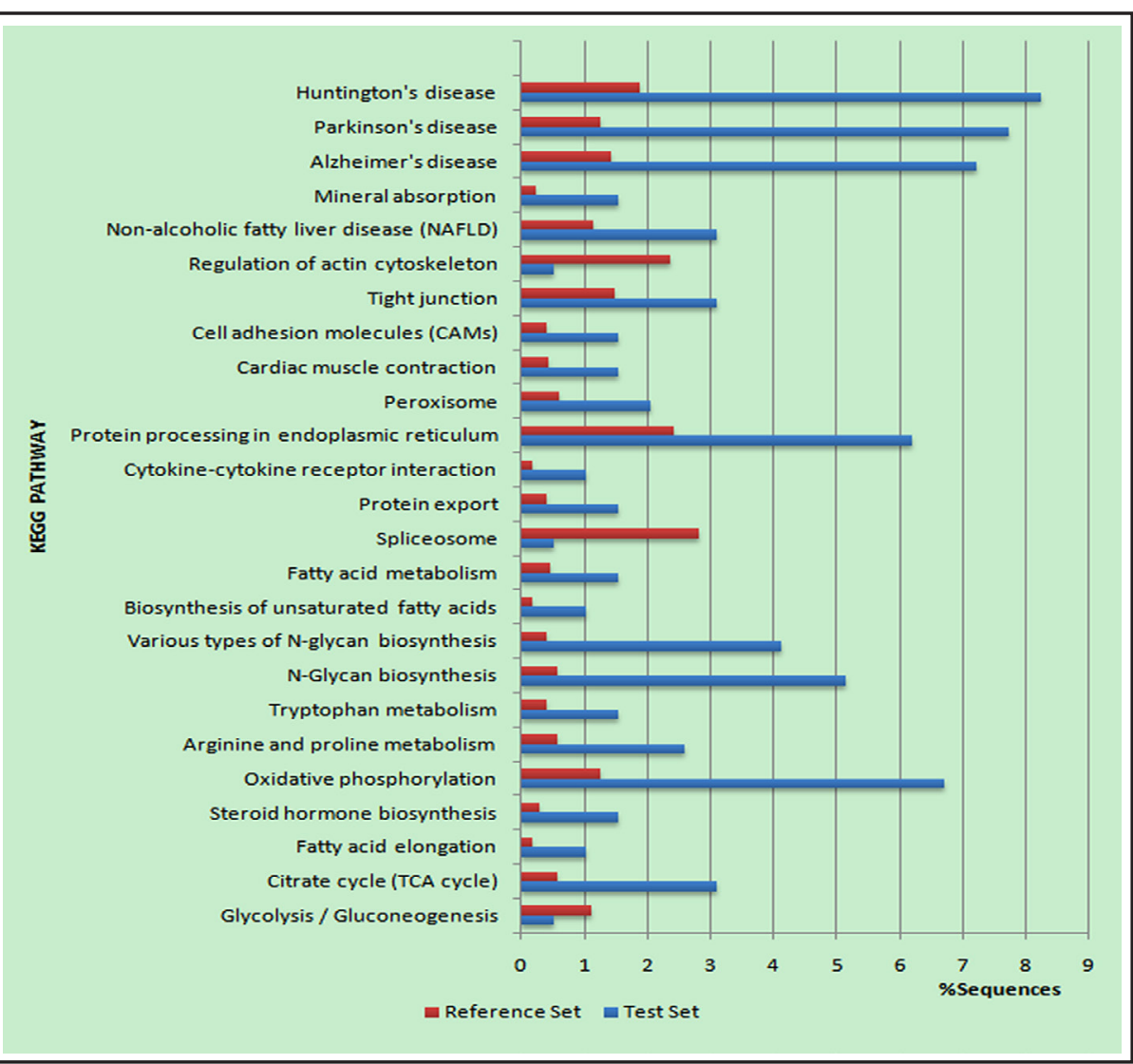

complex (64 proteins), membrane-enclosed lumen (34 proteins) and extracellular region (19 proteins), implying that most of the differential proteins were secretary proteins (Fig. 2A). The main biological process (BP) of these proteins were cellular process (156 proteins), singleorganism process (126 proteins), metabolic process (118 proteins), biological regulation (108 proteins), response to stimulus (77 proteins), multicellular organismal process (52 proteins), localization (51 proteins), signaling (47 proteins) and cellular component organization or biogenesis (46 proteins) (Fig. 2B). The main molecular function (MF) were binding (145 proteins), catalytic activity (77 proteins), transporter activity (15 proteins) and enzyme regulator activity (7 proteins) (Fig. 2C). Further GO enrichment demonstrated mitochondrion, transmembrane transport and transmembrane transporter activity were the most abundant categories in CC, BP and MF, respectively (Fig. 2D). Furthermore, there were at least 2 proteins (S100-A8, C4b-B) that correlated with inflammation, 1 protein (CD63) that correlated with coagulation, 3 proteins (ATP synthase subunit beta, cDNA FLJ14908 fis and endoglin) that correlated with angiogenesis/vascularization, and 11 proteins (interleukin-27 subunit beta, F11 receptor, isoform CRA_a, dynamin-2, protein SEC13 homolog, S100-A8, C4b-B, calnexin, serpin B9, stomatin-like protein 2, phosphoinositide 3-kinase adapter protein 1 and dolichyl-diphosphooligosaccharide-protein glycosyltransferase $48 \mathrm{kDa}$ subunit) that correlated with immunoregulation. All the aforementioned mentioned proteins were upregulated in sPE group.

When KEGG pathway analysis was performed on these differentially expressed proteins to evaluate which pathways were significantly represented, 151 pathways were found to match (data not shown). Furthermore, KEGG pathway enrichment revealed that 25 pathways were enriched, and that these differentially expressed proteins were mainly involved in glycolysis/gluconeogenesis, citrate cycle, fatty acid elongation, steroid hormone biosynthesis and oxidative phosphorylation, etc (Fig. 3). It is notable that some proteins were involved in pathways correlating with neurodegenerative diseases, such as Alzheimer's disease, Parkinson's disease and Huntington's disease. 


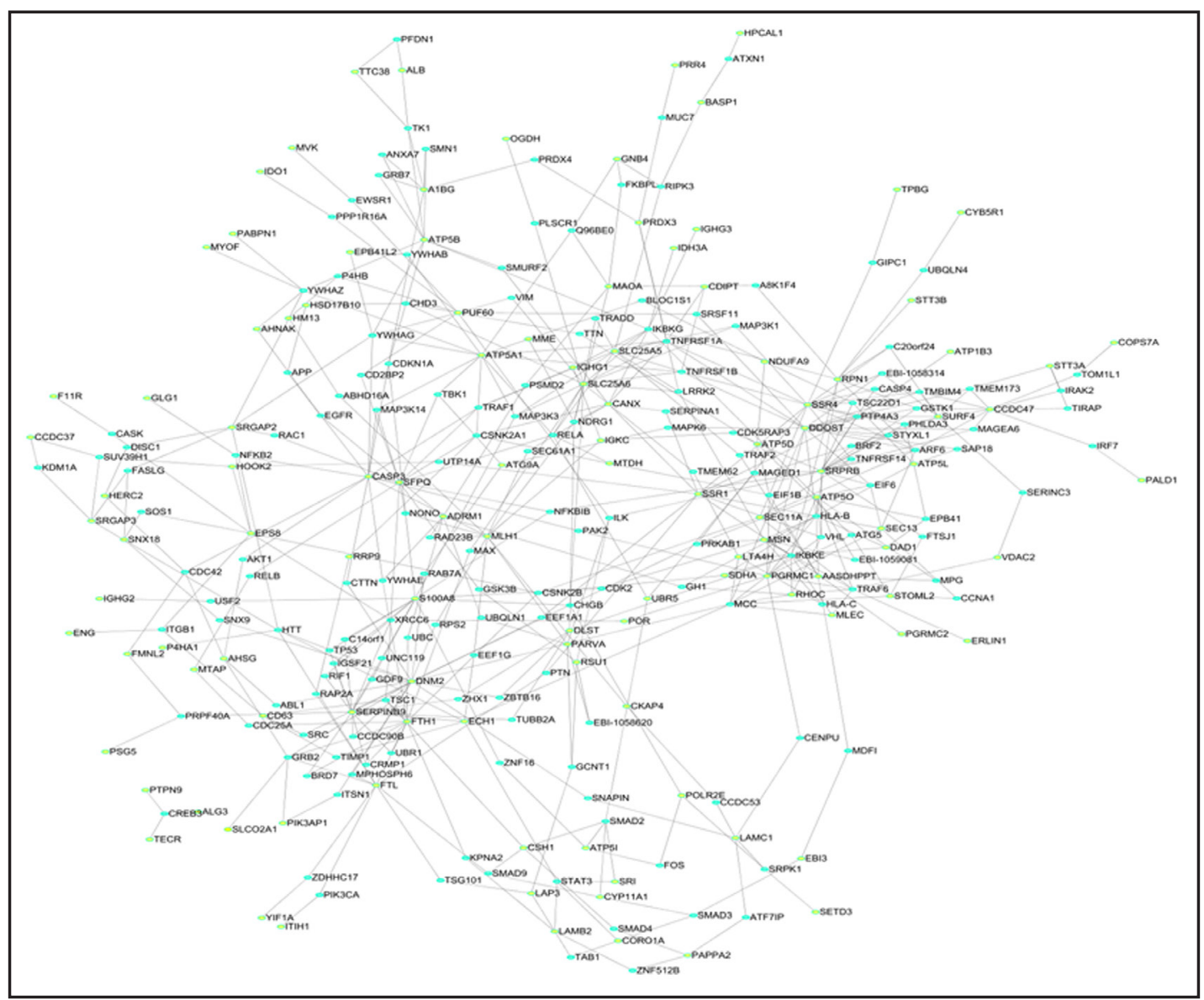

Fig. 4. The interacted network of differentially expressed proteins was analyzed by STRING (Search Tool for the Retrieval of Interacting Genes/Proteins) software. Differentially expressed proteins marked by yellow nodes and directly interacting proteins marked by blue nodes.

In addition, STRING database analysis revealed that most of these differentially expressed proteins were involved in physical or functional interaction to constitute a network (Fig. 4).

Hierarchical clustering was performed to detect the rationality and accuracy of selecting differentially expressed proteins, as well as group the data based on the degree of similarity and variability between the experiments and controls. As shown in Figure 5, the hierarchical clustering analysis classified the proteins into two major clusters, which separated upregulated and down- regulated proteins in each group. The resulting heatmap (Fig. 5) also shows a clustering of the samples coming from two different groups.

Validation of differentially expressed proteins identified by proteomics using western blotting

Four differentially expressed proteins (siglec-6, calnexin, CD63 and S100-A8) were chosen for western blotting (Fig. 6), according to the aforementioned bioinformatics analysis. Four proteins expressed high levels in the sPE group and showed the same trends as the iTRAQ results.

\section{Discussion}

Both eukaryotic and prokaryotic cells release extracellular vesicles (EVs) to their environment, which are spherical particles enclosed by a phospholipid bilayer and now 
Fig. 5. Hierarchical clustering of differentially expressed proteins. Clustering was based on protein expression levels in STBM from control and SPE group. Up-regulated protein expression values are displayed in red, and down-regulated values are in green.

thought to contribute to both physiology and pathology [32, 33]. Currently, there has no absolute consensus on classification, isolation and detection of EVs. The reported review indicated that based on their biogenesis, there are two main types of EVs have attracted substantial attention: exosomes and microvesicles [26, 34-36], as well as three purification protocols: differential centrifugation, density gradient separation and immunoaffinity capture methods [29, 37]. Each protocol has its limitations, for differential centrifugation, the final pellet is contaminated with cosedimenting vesicles and protein aggregates, vesicles with similar buoyant densities may co-sediment in density gradient separation and, although the immunoaffinity capture method has been reported to yield highquality exosome preparations [37], this method is based on a membrane antigen, meaning that only EVs positive for the antigen are acquired, whereas the negative population is excluded, and as the specific each type of EV is lacking, this method will again yield a mixed population, especially in body fluids. Differential centrifugation is the simplest and most commonly used protocol. Electron microscopy, flow cytometric analysis, western blotting and nanoparticle tracking analysis (NTA) were frequently used for detecting the size, morphology, concentration and origin of EVs.

$\mathrm{PE}$ is a heterogeneous disorder with two distinct subtypes: early- and late-onset membrane topography and antigen for

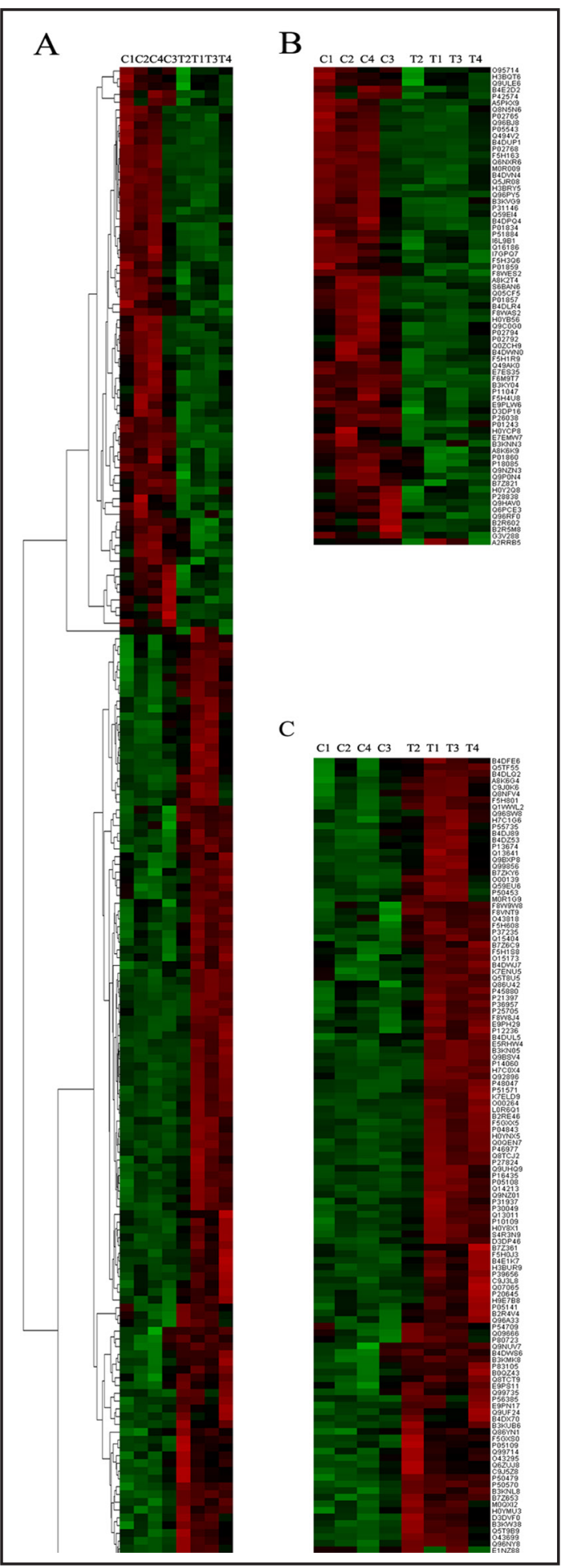

PE [1]. Although early-onset PE represents the minority of cases (about 10\%), it confers a high risk of life-threatening maternal complications and fetal death compared with lateonset PE. Women with early-onset PE are at increased risk for future cardiovascular disease. Children exposed to early-onset PE as a fetus also have heightened risk of high blood pressure, metabolic syndrome, and cardiovascular diseases at relative early age. Research studies should treat the 2 PE subtypes as distinct entities from etiological and prognostic aspects. Early-onset sPE is generally considered as a primarily placental disease, according 
A

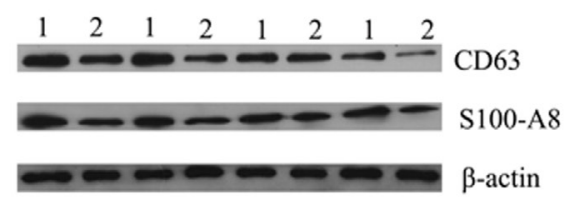

B

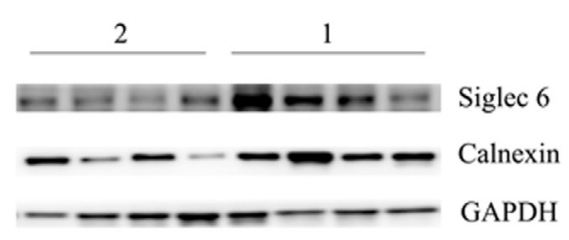

C

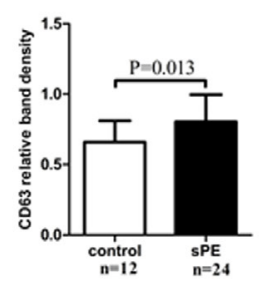

E

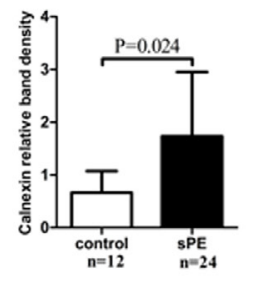

D

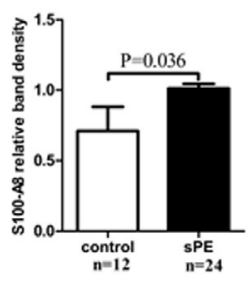

F

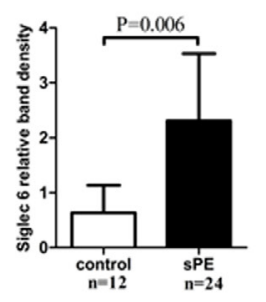

Fig. 6. Validation of the four differentially expressed proteins by western blot analysis. (A)-(B) Representative images of western blotting for CD63, S100-A8, siglec 6 and calnexin in the control (1) and sPE (2) group, respectively. (C)-(F) Histograms of expression levels (relative band density) for CD63, S100-A8, siglec 6 and calnexin in control $(n=12)$ and $\mathrm{SPE}(\mathrm{n}=24)$ group, respectively.

to a widely accepted "3-stage model" etiological hypothesis [4]. It is, in brief, characterized by abnormal immune tolerance (Stage 1), followed by incomplete placentation with reduced remodeling of maternal uteroplacental spiral arteries (Stage 2), and subsequently placental hypoxia-ischemia-induced placental factors released increasingly into the maternal circulation, leading to excessive systemic inflammation, endothelial dysfunction and the clinical signs of PE (Stage 3). However, there has no accurate biomarkers for diagnosis and therapy of this disease. Recently, extensive research on various types of EVs indicated that EVs may serve as potential clinical biomarkers for prognosis, diagnosis and therapy of certain diseases. For example, EVs from tumor antigen-pulsed dendritic cells (DCs) have been exploited for cancer immunotherapy, and EVs from mesenchymal stem cells (MSCs) have been used to stimulate tissue repair following myocardial infarction [32]. Thus, we can expect the potential application value of placenta-derived EVs on early diagnosis and clinical therapy for Early-onset sPE.

As an important placental factor, STBM was initially studied in normal pregnancy and PE by Redman et al. at the University of Oxford, then various research teams worldwide are dedicated to this area and have developed the nomenclature of STBM to include terms such as syncytiotrophoblast microvilli [6], syncytiotrophoblast microvillous membrane $[11,16,17,19]$, syncytiotrophoblast microparticles [15], syncytiotrophoblast microvesicles [23], syncytiotrophoblast-derived microparticles [8], and syncytiotrophoblast extracellular vesicles $[38,39]$, etc. Increasing evidence shows that STBM have various functions relevant to PE, but the precise mechanism is not fully explained. To well exploit the mechanism and biological function of STBM in early-onset SPE, further studies about its composition are required. Here, we analyzed the ingredients of STBM from the early-onset sPE patients by iTRAQ quantitative proteomics technology. We use the term 'syncytiotrophoblast extracellular vesicles' to refer to both syncytiotrophoblast microparticle and exosome vesicle types. Because STBM only account for less than $6 \%$ of the total number of EVs in the blood of normotensive pregnant women, it is very difficult to isolate pure and adequate amounts of 
STBM from maternal blood [40]. To our knowledge, based on the differential centrifugation protocol, there are four methods of generating STBM in vitro. STBM generated by explant culture (eSTBM), mechanical dissection (mSTBM) and placental perfusion (pSTBM) are from placentae [15], whereas the fourth source of STBM was from trophoblast-derived cell line (ATCC no. CRL-1584) [9]. Reported articles revealed that different preparation of STBM had similar morphology but various biological functions in the target cells [15, 23, 24]. However, due to the inaccessibility of deported trophoblasts in vivo, the overriding mechanism for STBM release and the status of STBM (apoptotic vs necrotic) were not determined. Both apoptosis and necrosis may be involved in the shedding of STBM, but the balance between apoptotic and necrotic shedding is presently unknown. Most researchers consider that eSTBM and pSTBM may more closely mimic the EVs released in physiological status. Both methods are widely used $[20,23,41,42]$. It has previously shown that the syncytiotrophoblast undergoes significant artefactual degradation during the first $24 \mathrm{~h}$ of villous explant culture [43]. By $72 \mathrm{~h}$ of culture, the underlying cytotrophoblast have fused to form a new syncytium that has formed under the syncytium in vitro $[43,44]$. This may explain that Redman et al. who only cultured $24 \mathrm{~h}$ in their explant culture model or used a short term perfusion model to isolate STBM, where the syncytium would have been more representative of that present in vivo. But Abumaree et al. also considered that the cultured time extend to 48 or $72 \mathrm{~h}$ in the explant culture model might be acceptable [43]. We adopted the eSTBM method combined with differential centrifugation, and the STBM preparations were verified by electron microscopy (EM) and western blot analysis. Finally, through 8-Plex iTRAQ labeling coupled with 2D nano LC-MS/MS, we identified 194 differentially expressed proteins in STBM from early-onset severe PE patients, 122 of which were up-regulated and 72 of which were downregulated. Furthermore, bioinformatics analysis showed that the main outcome was not well accordance with our previously supposed and other's preliminary results [26], as the differentially expressed proteins correlated with inflammatory, coagulation, angiogenesisor immunoregulation were not the salient in GO and KEGG pathway enrichment. We ascribed three reasons for these disparities. Firstly, this may be due to different proteomic methodologies, as we used 8-Plex iTRAQ labeling coupled with 2D nano LC-MS/MS, whereas Redman's team used multi-dimensional protein identification technology (MudPIT) and ultra-performance liquid chromatography tandem mass spectrometry analysis (UPLC-MSE). Secondly, the preparation of STBM may have played a role, although both of the STBM were prepared from placentae, the explicit method was different, we employed eSTBM whereas they used pSTBM. It should be noted that both methods are widely accepted. Our results may indicate that eSTBM carried different repertoire of molecules with pSTBM. The third aspect may due to the selection of patients, as we focused on the early-onset severe subtype of PE, whereas their selection of patients was not designated, as severe and moderate PE, earlyonset and late-onset PE may have different etiologies and pathogenesis.

Our analysis also reveals that the differentially expressed protein, siglec-6, which has been proven only expressed in human placenta [45] was the most up-regulated protein (fold-change $=4.091$ ). Recently, Rumer, etal. [46] indicated that siglec-6 was increased in both the basal plate and chorionic villi of preterm PE placenta. Inadequate invasion of trophoblast may cause PE [47]. Lam's [48] results showed that siglec-6 may interact with glycodelin-A (GdA) to suppress trophoblast invasiveness by down-regulating the ERK/ c-Jun signaling pathway. Our results further enhance the linkage between siglec- 6 and the earlyonset severe subtype of PE. Increased shedding of siglec- 6 carried by STBM may be part of the pathologic processes in PE, especially in early-onset SPE. The specific mechanism need to be clarified in future studies.

Although it has not been enriched, we also acquired some differentially expressed proteins with inflammation, coagulation, angiogenesis and immunoregulation. We cannot exclude the possibility that these proteins may be triggers and have certain capacity to switch on or exaggerate the role of key effector molecules, and further studies should be carried out.

We performed an ITRAQ quantitative proteomics analysis of eSTBM from the earlyonset sPE patients for the first time. This may be a fundamental opening for us to exploit 
the specific mechanisms and functions of STBM in PE. However, the eSTBM prepared in our study were also mixture because of the lack of a gold standard for the classification, isolation and detection of EVs. We could not tell whether the identified proteins were from microparticles or exosomes. Because they have different biological functions, cargos and modes of production, many methods $[49,50]$ have been used to detect and to discriminate microparticles and exosomes. Some novel commercially available technologies, such as nanoparticle tracking analysis (NTA) and resistive pulse sensing (RPS), have also arisen.

\section{Conclusions}

In this study, we performed a proteomic analysis on STBM derived from early-onset sPE patients, using iTRAQ isobaric tags and 2D nano LC-MS/MS. We identified 194 differentially expressed proteins in STBM derived from early-onset SPE patients. Further bioinformatics analysis revealed that mitochondrion, transmembrane transport and transmembrane transporter activity were the most abundant categories in CC, BP and MF, respectively. Glycolysis/gluconeogenesis, citrate cycle, fatty acid elongation, steroid hormone biosynthesis and oxidative phosphorylation were the five significantly represented pathways. Our results provide fundamental opening and evidence for future studies, which are needed to elucidate the specific mechanisms of the differentially expressed proteins with inflammation, coagulation, angiogenesis and immunoregulation in early-onset sPE.

\section{Acknowledgments}

This work was supported by grants from the National Natural Science Foundation of China (No. 81170576, No. 31470886 and No. 81270008), the application program of Chongqing Science and Technology Committee, China (No. 20ByykfA0213).

\section{Disclosure Statement}

The authors declare that they have no conflict of interest.

\section{References}

1 Steegers EA, von Dadelszen P, Duvekot JJ, Pijnenborg R: Pre-eclampsia. Lancet 2010;376:631-644.

2 Cunningham FG, Leveno KJ, Bloom SL, Hauth JC, Rouse DJ, Spong CY: Pregnancy hypertension; in Cunningham FG, Leveno KJ, Bloom SL, Hauth JC, Rouse DJ, Spong CY (eds): Williams Obstetrics 23rd ed. New York, The McGraw-Hill Companies, 2010, pp987-1046.

3 Staff AC, Benton SJ, von Dadelszen P, Roberts JM, Taylor RN, Powers RW, Charnock-Jones DS, Redman CW: Redefining preeclampsia using placenta-derived biomarkers. Hypertension 2013;61:932-942.

4 Redman CW, Sargent IL: Immunology of pre-eclampsia. Am J Reprod Immunol 2010;63:534-543.

5 Liu L, Zhang X, Rong C, Rui C, Ji H, Qian YJ, Jia R, Sun L: Distinct DNA methylomes of human placentas between pre-eclampsia and gestational diabetes mellitus. Cell Physiol Biochem 2014;34:1877-1889.

6 Knight M, Redman CW, Linton EA, Sargent IL: Shedding of syncytiotrophoblast microvilli into the maternal circulation in pre-eclamptic pregnancies. Br J Obstet Gynaecol 1998;105:632-640.

7 Goswami D, Tannetta DS, Magee LA, Fuchisawa A, Redman CW, Sargent IL, von Dadelszen P: Excess syncytiotrophoblast microparticle shedding is a feature of early-onset pre-eclampsia, but not normotensive intrauterine growth restriction. Placenta 2006;27:56-61.

8 Chen Y, Huang Y, Jiang R, Teng Y: Syncytiotrophoblast-derived microparticle shedding in early-onset and late-onset severe pre-eclampsia. Int J Gynaecol Obstet 2012;119:234-238. 


\section{Cellular Physiology Cell Physiol Biochem 2015;36:1116-1130 \begin{tabular}{l|l|l}
\hline DOI: 10.1159/000430283 & (C) 2015 S. Karger AG, Basel
\end{tabular} and Biochemistry Published online: June 25, 2015

Li et al.: Proteomic Analysis of Syncytiotrophoblast Extracellular Vesicles

9 Lee SM, Romero R, Lee YJ, Park IS, Park CW, Yoon BH: Systemic inflammatory stimulation by microparticles derived from hypoxic trophoblast as a model for inflammatory response in preeclampsia. Am J Obstet Gynecol 2012;207:337 e331-338.

10 Holder BS, Tower CL, Jones CJ, Aplin JD, Abrahams VM: Heightened pro-inflammatory effect of preeclamptic placental microvesicles on peripheral blood immune cells in humans. Biol Reprod 2012;86:103.

11 Aly AS, Khandelwal M, Zhao J, Mehmet AH, Sammel MD, Parry S: Neutrophils are stimulated by syncytiotrophoblast microvillous membranes to generate superoxide radicals in women with preeclampsia. Am J Obstet Gynecol 2004;190:252-258.

12 Germain SJ, Sacks GP, Sooranna SR, Sargent IL, Redman CW: Systemic inflammatory priming in normal pregnancy and preeclampsia: The role of circulating syncytiotrophoblast microparticles. J Immunol 2007;178:5949-5956.

13 Gardiner C, Tannetta DS, Simms CA, Harrison P, Redman CW, Sargent IL: Syncytiotrophoblast microvesicles released from pre-eclampsia placentae exhibit increased tissue factor activity. PLoS One 2011;6:e26313.

14 Guller S, Tang Z, Ma YY, Di Santo S, Sager R, Schneider H: Protein composition of microparticles shed from human placenta during placental perfusion: Potential role in angiogenesis and fibrinolysis in preeclampsia. Placenta 2011;32:63-69.

15 Gupta AK, Rusterholz C, Huppertz B, Malek A, Schneider H, Holzgreve W, Hahn S: A comparative study of the effect of three different syncytiotrophoblast micro-particles preparations on endothelial cells. Placenta 2005;26:59-66.

16 Smarason AK, Sargent IL, Starkey PM, Redman CW: The effect of placental syncytiotrophoblast microvillous membranes from normal and pre-eclamptic women on the growth of endothelial cells in vitro. Br J Obstet Gynaecol 1993;100:943-949.

17 Cockell AP, Learmont JG, Smarason AK, Redman CW, Sargent IL, Poston L: Human placental syncytiotrophoblast microvillous membranes impair maternal vascular endothelial function. Br J Obstet Gynaecol 1997;104:235-240.

18 Kertesz Z, Linton EA, Redman CW: Adhesion molecules of syncytiotrophoblast microvillous membranes inhibit proliferation of human umbilical vein endothelial cells. Placenta 2000;21:150-159.

19 Hoegh AM, Tannetta D, Sargent I, Borup R, Nielsen FC, Redman C, Sorensen S, Hviid TV: Effect of syncytiotrophoblast microvillous membrane treatment on gene expression in human umbilical vein endothelial cells. BJOG 2006;113:1270-1279.

20 Gupta AK, Holzgreve W, Hahn S: Decrease in lipid levels of syncytiotrophoblast micro-particles reduced their potential to inhibit endothelial cell proliferation. Arch Gynecol Obstet 2008;277:115-119.

21 Lok CA, Snijder KS, Nieuwland R, Van Der Post JA, de Vos P, Faas MM: Microparticles of pregnant women and preeclamptic patients activate endothelial cells in the presence of monocytes. Am J Reprod Immunol 2012;67:206-215.

22 Sabapatha A, Gercel-Taylor C, Taylor DD: Specific isolation of placenta-derived exosomes from the circulation of pregnant women and their immunoregulatory consequences. Am J Reprod Immunol 2006;56:345-355.

23 Southcombe J, Tannetta D, Redman C, Sargent I: The immunomodulatory role of syncytiotrophoblast microvesicles. PLoS One 2011;6:e20245.

24 Gupta AK, Rusterholz C, Holzgreve W, Hahn S: Syncytiotrophoblast micro-particles do not induce apoptosis in peripheral t lymphocytes, but differ in their activity depending on the mode of preparation. J Reprod Immunol 2005;68:15-26.

25 Mincheva-Nilsson L, Baranov V: Placenta-derived exosomes and syncytiotrophoblast microparticles and their role in human reproduction: Immune modulation for pregnancy success. Am J Reprod Immunol 2014;72:440-457.

26 Redman CW, Tannetta DS, Dragovic RA, Gardiner C, Southcombe JH, Collett GP, Sargent IL: Review: Does size matter? Placental debris and the pathophysiology of pre-eclampsia. Placenta 2012;33:S48-54.

27 Messerli M, May K, Hansson SR, Schneider H, Holzgreve W, Hahn S, Rusterholz C: Feto-maternal interactions in pregnancies: Placental microparticles activate peripheral blood monocytes. Placenta 2010;31:106-112.

28 Smith NC, Brush MG, Luckett S: Preparation of human placental villous surface membrane. Nature 1974;252:302-303. 


\section{Cellular Physiology Cell Physiol Biochem 2015;36:1116-1130 \begin{tabular}{l|l|l}
\hline DOI: 10.1159/000430283 & (C) 2015 S. Karger AG, Basel
\end{tabular} and Biochemistry Published online: June 25, 2015

Li et al.: Proteomic Analysis of Syncytiotrophoblast Extracellular Vesicles

29 Théry C, S. Amigorena, G. Raposo, and A. Clayton.: Isolation and characterization of exosomes from cell culture supernatants and biological fluids. Current Protocols in Cell Biology 2006; Chapter 3:Unit 3.22

30 Unwin RD, Griffiths JR, Whetton AD: Simultaneous analysis of relative protein expression levels across multiple samples using itraq isobaric tags with 2d nano lc-ms/ms. Nat Protoc 2010;5:1574-1582.

31 Wisniewski JR, Zougman A, Nagaraj N, Mann M: Universal sample preparation method for proteome analysis. Nat Methods 2009;6:359-362.

32 S ELA, Mager I, Breakefield XO, Wood MJ: Extracellular vesicles: Biology and emerging therapeutic opportunities. Nat Rev Drug Discov 2013;12:347-357.

33 Tsai WH, Shih CH, Feng SY, Li IT, Chang SC, Lin YC, Hsu HC: Cx3cl1(+) microparticles mediate the chemoattraction of alveolar macrophages toward apoptotic acute promyelocytic leukemic cells. Cell Physiol Biochem 2014;33:594-604.

34 van der Pol E, Boing AN, Harrison P, Sturk A, Nieuwland R: Classification, functions, and clinical relevance of extracellular vesicles. Pharmacol Rev 2012;64:676-705.

35 Cocucci E, Racchetti G, Meldolesi J: Shedding microvesicles: Artefacts no more. Trends Cell Biol 2009;19:4351.

36 Raposo G, Stoorvogel W: Extracellular vesicles: Exosomes, microvesicles, and friends. J Cell Biol 2013;200:373-383.

37 Tauro BJ, Greening DW, Mathias RA, Ji H, Mathivanan S, Scott AM, Simpson RJ: Comparison of ultracentrifugation, density gradient separation, and immunoaffinity capture methods for isolating human colon cancer cell line lim1863-derived exosomes. Methods 2012;56:293-304.

38 Dragovic RA, Southcombe JH, Tannetta DS, Redman CW, Sargent IL: Multicolor flow cytometry and nanoparticle tracking analysis of extracellular vesicles in the plasma of normal pregnant and pre-eclamptic women. Biol Reprod 2013;89:151.

39 Cronqvist T, Salje K, Familari M, Guller S, Schneider H, Gardiner C, Sargent IL, Redman CW, Morgelin M, Akerstrom B, Gram M, Hansson SR: Syncytiotrophoblast vesicles show altered micro-rna and haemoglobin content after ex-vivo perfusion of placentas with haemoglobin to mimic preeclampsia. PLoS One 2014;9:e90020.

40 Aharon A, Brenner B: Microparticles and pregnancy complications. Thromb Res 2011;127 Suppl 3:S67-71.

41 Rusterholz C, Holzgreve W, Hahn S: Oxidative stress alters the integrity of cell-free mrna fragments associated with placenta-derived syncytiotrophoblast microparticles. Fetal Diagn Ther 2007;22:313-317.

42 Tannetta DS, Dragovic RA, Gardiner C, Redman CW, Sargent IL: Characterisation of syncytiotrophoblast vesicles in normal pregnancy and pre-eclampsia: Expression of flt-1 and endoglin. PLoS One 2013;8:e56754.

43 Abumaree MH, Stone PR, Chamley LW: An in vitro model of human placental trophoblast deportation/ shedding. Mol Hum Reprod 2006;12:687-694.

44 Siman CM, Sibley CP, Jones CJ, Turner MA, Greenwood SL: The functional regeneration of syncytiotrophoblast in cultured explants of term placenta. Am J Physiol Regul Integr Comp Physiol 2001;280:R1116-1122.

45 Brinkman-Van der Linden EC, Hurtado-Ziola N, Hayakawa T, Wiggleton L, Benirschke K, Varki A, Varki N: Human-specific expression of siglec-6 in the placenta. Glycobiology 2007;17:922-931.

46 Rumer KK, Uyenishi J, Hoffman MC, Fisher BM, Winn VD: Siglec-6 expression is increased in placentas from pregnancies complicated by preterm preeclampsia. Reprod Sci 2013;20:646-653.

47 Jia RZ, Ding GC, Gu CM, Huang T, Rui C, Wang YX, Lu Q: Cdx2 enhances htr-8/svneo trophoblast cell invasion by altering the expression of matrix metalloproteinases. Cell Physiol Biochem 2014;34:628-636.

48 Lam KK, Chiu PC, Lee CL, Pang RT, Leung CO, Koistinen H, Seppala M, Ho PC, Yeung WS: Glycodelin-a protein interacts with siglec-6 protein to suppress trophoblast invasiveness by down-regulating extracellular signal-regulated kinase (erk)/c-jun signaling pathway. J Biol Chem 2011;286:37118-37127.

49 van der Pol E, Coumans F, Varga Z, Krumrey M, Nieuwland R: Innovation in detection of microparticles and exosomes. J Thromb Haemost 2013;11 Suppl 1:36-45.

50 van der Pol E, Hoekstra AG, Sturk A, Otto C, van Leeuwen TG, Nieuwland R: Optical and non-optical methods for detection and characterization of microparticles and exosomes. J Thromb Haemost 2010;8:2596-2607. 